\title{
LETTER OPEN \\ Impact of mechanical circulatory support and immunomodulation therapy on outcome of patients with fulminant myocarditis: Chinese registry of fulminant myocarditis
}

Signal Transduction and Targeted Therapy (2021)6:350

\section{Dear Editor,}

Patients presenting with acute myocarditis and sudden hemodynamic instability (termed fulminant myocarditis [FM]) still have a high mortality and need for heart transplantation, up to $28 \%$ at 60 days. $^{1-3}$ Recent scientific statements and expert opinion consensus suggests early use of temporary mechanical circulatory supports (t-MCS)., Specifically, Chinese scientific statement proposed an extensive use of t-MCS combined with immunoregulatory therapy (IT), ${ }^{4}$ although formal trials are lacking. We present a multicenter, retrospective study to compare the outcome of patients who were treated with t-MCS and IT vs. patients who didn't receive these treatments. We included patients with the diagnosis of FM based on the presence of viral prodromal signs/ symptoms followed by acute onset of severe heart failure (HF) without other relevant differential diagnosis or pre-existing cardiac disorders. Patients who received both t-MCS and IT during hospitalization were classified as t-MCS+IT group. T-MCS used were intra-aortic balloon pulsation (IABP) (median duration 7 days, first to third quartile [Q1-Q3, 4-8]) or venous-arterial extracorporeal membrane oxygenation (VA-ECMO) (median duration 5 days [Q1-Q3, 5-7], or both. IT included methylprednisolone (200-400 mg) or dexamethasone (20-40 mg), qd for 3-5 days of intravenous (IV) and then gradually down titrated and weaned in 7-10 days, and IV immunoglobulin (10-20 g qd for the 3-5 days and then $10 \mathrm{~g}$ for another 3-5 days). Patients who didn't receive both t-MCS and IT were classified as non-t-MCS+IT 3 group. Statistical differences were analyzed using the Mann-Whitney $U$ test for continuous variables. Categorical variables were compared using Fisher's exact test or $x^{2}$ test.

A total of 138 FM patients with a median age of 33 years (Q1-Q3, 26-41) and a male prevalence of $55.1 \%$ were included in the study (Fig. 1a, Supplementary Table 1). Among 138 patients, $96(69.6 \%)$ patients received $\mathrm{t}-\mathrm{MCS}+\mathrm{IT}$ during hospitalization (median age 32 years [Q1-Q3, 26-44]; 55.2\% men). Forty-two patients (30.4\%) were in the non-t-MCS+IT group (median age 33 years [Q1-Q3, 25-42]; 54.8\% men), among them 16 patients received only t-MCS, 17 patients received only IT and 9 patients received neither t-MCS nor IT. The two groups of FM patients didn't differ for cardiovascular risk factors, systolic blood pressure $(\mathrm{SBP})$, heart rate $(\mathrm{HR})$, clinical presentation, laboratory findings, and presence of severe ventricular arrythmias (VAs) except for a higher prevalence of fever in t-MCS+IT group compared with non$\mathrm{t}-\mathrm{MCS}+\mathrm{IT}(74.0 \%$ vs. $42.9 \%$ respectively, $p<0.001)$.

Overall, in-hospital mortality of FM patients was $18.8 \%$ (26 out of 138). We observed $4.2 \%$ deaths (4 of 96 patients) in the t-MCS +IT group vs. $52.4 \%$ (22 of 42 patients) in the non-t-MCS+IT group

\author{
; https://doi.org/10.1038/s41392-021-00700-6
}

(Unadjusted OR 0.03, 95\% confidence interval [Cl] 0.13-0.92, $p=$ 0.001) (Supplementary Table 2). Both t-MCS and IT contributed to the reduction of mortality, however, separate use of t-MCS and IT were associated with a higher mortality compared to combined t-MCS and IT (Supplementary Table 2). Neither heart transplantation nor longterm left ventricular assist device implant was performed. In-hospital mortality risk of FM patients was reduced by $92.7 \%$ in $\mathrm{t}-\mathrm{MCS}+\mathrm{IT}$ group (Fig. 1b). Multiorgan failure due to irreversible cardiogenic shock was the cause of all deaths. In the logistic regression model after adjusting for age, gender, and inotrope agents, the use of t-MCS + IT was associated with lower all-cause mortality (adjusted OR, $0.11 ; 95 \% \mathrm{Cl}, 0.09-0.46 ; p=0.001$ ) vs non-t-MCS+IT group (Supplementary Table 3). Patients with VAs and/or advanced atrioventricular block (AVB) were found to be more beneficial from t-MCS + IT (adjusted OR 0.08, 95\% Cl 0.01-0.62; adjusted OR 0.32, 95\% $\mathrm{Cl}$ 0.10-0.996, respectively). Among the survivors, t-MCS+IT and non-t-MCS+IT patients had similar hospital stay (Fig. 1c).

Apart from the different use of t-MCS and IT, in the non-t-MCS + IT group there was a significant higher proportion of patients on inotropic agents, whereas invasive ventilation and continuous veno-venous hemodialysis were used only in the t-MCS+IT group (Supplementary Table 4).

After $30 \mathrm{~min}$ since institution of t-MCS, we observed a significant improvement in SBP compared to baseline. Patients in the t-MCS+IT showed higher SBP in the first 7 days compared to the non-t-MCS+IT (Fig. 1d). Left ventricular ejection fraction (LVEF) was improved more in the t-MCS+IT group compared with non-MCS+IT group, from a median LVEF of $40 \%$ at baseline to $57 \%$ in t-MCS+IT survivors (Fig. 1e). Furthermore, troponin I levels dropped more rapidly in the t-MCS + IT group compared with nont-MCS+IT group (Fig. 1f).

In summary, we observed that the use of t-MCS plus IT was associated with lower risk of death in FM patients. T-MCS allows patients to tide over the crisis, combined IT can wane myocardial inflammation, which together contributed to the striking reduction of in-hospital mortality of FM patients. A study limitation is that no histologic findings confirmed the clinical diagnosis of FM which is recommended as a most important diagnostic criteria of $\mathrm{FM}_{1}^{5}$ even if the clinical presentation and the course are typical, thus alternative diagnosis are unlikely. Our findings support the importance of combined application of t-MCS and IT in the treatment of FM, although more confirmative clinical evidence are needed.

\section{DATA AVAILABILITY}

The data are available from the corresponding author on reasonable request. 

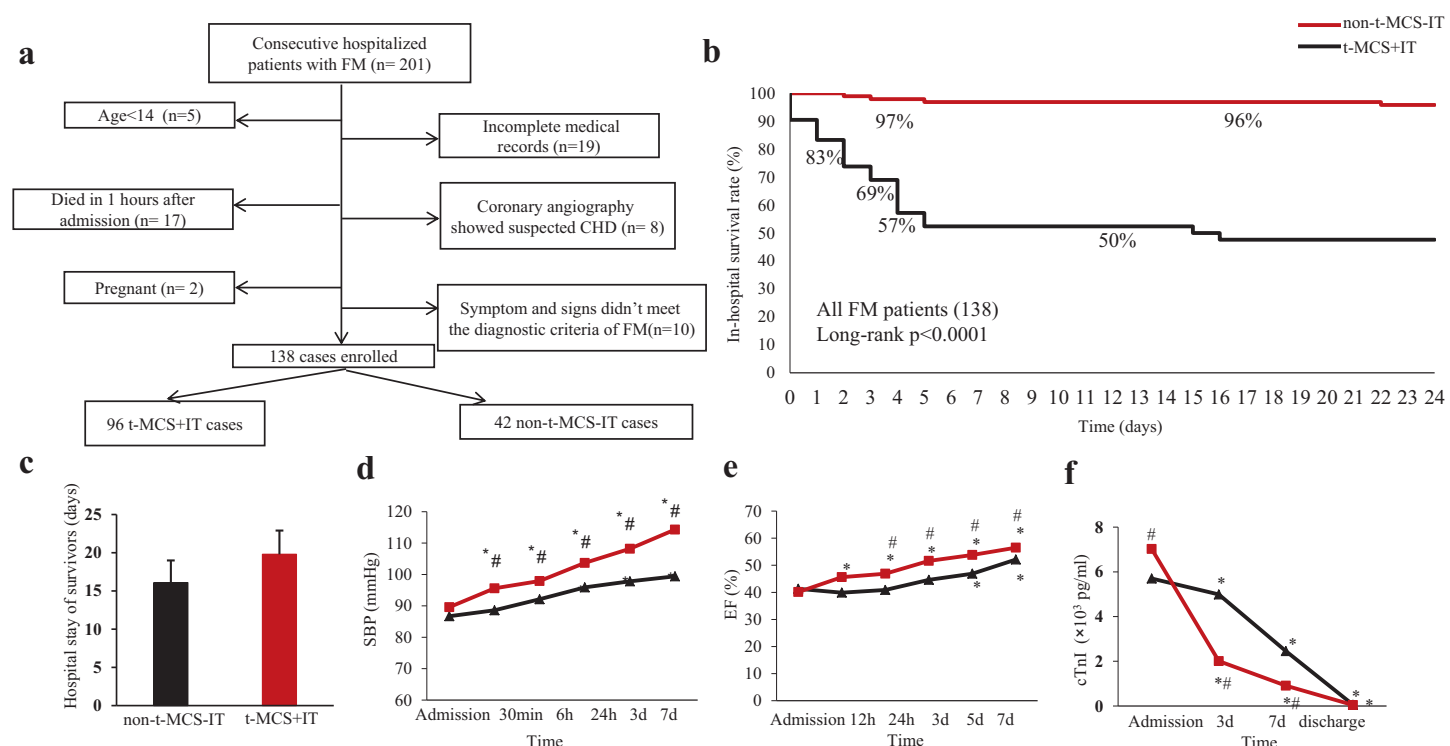

Fig. 1 a Flow diagram describing the selection of 138 subjects from the overall population diagnosed as FM. b The Kaplan-Meier curves of FM patients treated by different methods. c The hospital stays length of survived FM patients in both groups. $\mathbf{d}$ The changes of systolic blood pressure (SBP). ${ }^{*} p<0.05$ vs baseline. ${ }^{\#} p<0.05$ vs non-t-MCS+IT group. e The changes of ejection fraction of left ventricle. $\mathbf{f}$ The changes of troponin I of FM patents. ${ }^{*} p<0.05$ vs baseline. ${ }^{\#} p<0.05$ vs non-t-MCS+IT group

\section{ACKNOWLEDGEMENTS}

This work grants from the National Natural Science Foundation of China (No. 81790624 and 81630010,82070316 ) and National Key R\&D Program of China (NO. 2017YFC0909400).

\section{ADDITIONAL INFORMATION}

Supplementary information The online version contains supplementary material available at https://doi.org/10.1038/s41392-021-00700-6.

Competing interests: No author had potential conflicts of interest involving the work under consideration for publication. E.A. received honoraria for participation to advisory board from Kiniksa Pharmaceutical in the last 3 years. Dao Wen Wang is the editorial board member of Signal Transduction and Targeted Therapy, but he has not been involved in the process of the manuscript handling.

Ning Zhou ${ }^{1}$, Yuhua Zhao ${ }^{2}$, Jiangang Jiang ${ }^{1}$, Lan Shen ${ }^{3}$, Junming $\mathrm{Li}^{4}$, Jing Wan ${ }^{5}$, Xueping Ma ${ }^{6}$, Jing Zhang ${ }^{7 凶 '}$ Enrico Ammirati ${ }^{8 凶}$ and Dao Wen Wang ${ }^{1 \times}$

${ }^{1}$ Division of Cardiology, Department of Internal Medicine, Tongji Hospital, Tongji Medical College, Huazhong University of Science and Technology, and Hubei Key Laboratory of Genetics and Molecular Mechanisms of Cardiological Disorders, Wuhan, China; ${ }^{2}$ Division of Cardiology, Dongguan Kanghua Hospital, Dongguan, China;

${ }^{3}$ Division of Cardiology, Shanghai Chest Hospital, Shanghai Jiao Tong University, Shanghai, China; ${ }^{4}$ Division of Cardiology, The First Hospital of Yichang City, Yichang, China; ${ }^{5}$ Division of Cardiology, Zhongnan Hospital of Wuhan University, Wuhan, China; ${ }^{6}$ Division of Cardiology, General Hospital of Ningxia Medical University, Yinchuan, China; 'Division of Cardiology, Henan Provincial People's Hospital, Zhengzhou, China and "De Gasperis" Cardio Center and Transplant Center, ASST Grande Ospedale Metropolitano Niguarda, Milan, Italy
Correspondence: Jing Zhang (zhangjingxnk@qq.com) or Enrico Ammirati (enrico.ammirati@ospedaleniguarda.it) or Dao Wen Wang (dwwang@tjh.tjmu.edu.cn)

\section{REFERENCES}

1. Kociol, R. D. et al. Recognition and initial management of fulminant myocarditis: a scientific statement from the American Heart Association. Circulation 141, e69-e92 (2020).

2. Ammirati, E. et al. Fulminant versus acute nonfulminant myocarditis in patients with left ventricular systolic dysfunction. J. Am. Coll. Cardiol. 74, 299-311 (2019).

3. Ammirati, E. et al. Management of acute myocarditis and chronic inflammatory cardiomyopathy: an expert consensus document. Circ. Heart Fail. 13, e007405 (2020).

4. Wang, D. et al. Chinese society of cardiology expert consensus statement on the diagnosis and treatment of adult fulminant myocarditis. Sci. China Life Sci. 62, 187-202 (2019).

5. Caforio, AlidaL. P. et al. Current state of knowledge on aetiology, diagnosis, management, and therapy of myocarditis: a position statement of the European Society of Cardiology Working Group on Myocardial and Pericardial Diseases. Eur. Heart J. 34, 2636-2648 (2013).

(i) Open Access This article is licensed under a Creative Commons Attribution 4.0 International License, which permits use, sharing, adaptation, distribution and reproduction in any medium or format, as long as you give appropriate credit to the original author(s) and the source, provide a link to the Creative Commons license, and indicate if changes were made. The images or other third party material in this article are included in the article's Creative Commons license, unless indicated otherwise in a credit line to the material. If material is not included in the article's Creative Commons license and your intended use is not permitted by statutory regulation or exceeds the permitted use, you will need to obtain permission directly from the copyright holder. To view a copy of this license, visit http://creativecommons. org/licenses/by/4.0/.

(c) The Author(s) 2021 